The University of San Francisco

USF Scholarship: a digital repository @ Gleeson Library |

Geschke Center

$11-9-1992$

\title{
Novel Results for Quasiclassical Linear Transport in Metallic Multilayers
}

Horacio E. Camblong

University of San Francisco, camblong@usfca.edu

Follow this and additional works at: http://repository.usfca.edu/phys

Part of the Physics Commons

\section{Recommended Citation}

Camblong, Horacio E., "Novel Results for Quasiclassical Linear Transport in Metallic Multilayers" (1992). Physics and Astronomy. Paper 3.

http://repository.usfca.edu/phys/3 


\title{
Novel Results for Quasiclassical Linear Transport in Metallic Multilayers
}

\author{
Horacio E. Camblong and Peter M. Levy \\ Department of Physics, New York University, New York, New York 10003
}

(Received 27 July 1992)

\begin{abstract}
We analyze the linear transport behavior of metallic multilayers via the Kubo formula for a Hamiltonian with zero-range spin-dependent potentials. We find a direct connection between the Boltzmann and the Kubo approaches. Our two-point transport theory validates the quasiclassical approach for multilayered structures and introduces a new treatment of interfaces via angle-dependent coherent transmission coefficients.
\end{abstract}

PACS numbers: $72.15 . \mathrm{Gd}, 73.50 . \mathrm{Bk}, 73.50 . \mathrm{Jt}$

Recently, considerable attention has been drawn by the discovery of negative giant magnetoresistance (GMR) in magnetic multilayers [1]. This new phenomenon is due to the magnetic-field-induced parallel reorientation of antiferromagnetically coupled magnetic layers across nonmagnetic spacers, rather than to the alteration of the electron's dynamics. The underlying mechanism for the decrease in the resistivity from antiparallel to parallel alignment is spin-dependent scattering [1]. Two conceptually different transport theories have been developed to account for the observed GMR: quasiclassical, based on the Boltzmann equation, and quantum, based on the Kubo formula. The quasiclassical approach, an extension of the Fuchs-Sondheimer theory [2], was first applied to multilayers by Carcia and Suna [3] and to magnetic superlattices by Camley and Barnás [4]. The quantum approach [5] starts from a model Hamiltonian and uses the Kubo formula, which provides the correct quantum-statistical calculation of the linear response coefficients [6]. Moreover, it is well known that the quasiclassical approach apparently fails to account simultaneously for the observed values of resistivity and magnetoresistance: it seems to underestimate the contribution of interfacial scattering $[5,7]$. However, one should not conclude prematurely that the quasiclassical theory fails for magnetic superlattices; as pointed out by Johnson and Camley [8], the problem mentioned above could be removed by treating interfacial scattering more realistically in terms of "mixing interlayers."

So far, no paper has formulated in a fundamental way (that is, using the Kubo formula) the following questions: whether or not the quasiclassical approach is applicable to metallic multilayers and, if the answer were affirmative, what the explicit connection between the quasiclassical and quantum approaches would be.

In this Letter, we present a nonlocal linear transport theory of metallic multilayers whereby we answer these fundamental questions. Our theory lends support to the quasiclassical approach, establishes the explicit connection between the two approaches, and, what is most important, introduces a new treatment of interface scattering. Our starting point is the model Hamiltonian of
Ref. [5], which we use together with the Kubo formula in real space to provide a direct comparison with the approach based on the Boltzmann equation. Our derivation is similar to the calculation of the bulk electrical resistivity of a homogeneous metallic solid at low temperatures, which is also governed by scattering by impurities and defects [9]; moreover, we use the following two basic ingredients: the quasiclassical character of transport in metallic multilayers (as defined below) and a partial restoration of translational invariance in the plane of the layers, via an "impurity average" (also discussed below).

We define the quasiclassical limit as the absence of quantum corrections of two types: quantum size effects and quantum interference corrections. Quantum size effects arise from the confinement of electrons with Fermi wave number $k_{F}$ in a finite well of size $L$ and their relative importance is measured by the parameter $k_{F} L$. Quantum interference effects arise from the interference of electron paths and play a fundamental role, in three dimensions, when $k_{F}^{-1} \gtrsim l$, where $l$ is the electron's mean free path. However, for metallic systems in general and metallic multilayers in particular, the relatively small value of $k_{F}^{-1}$ makes the quasiclassical limit an excellent approximation for transport properties, except for the cases of very dirty samples for which the mean free path becomes comparable to $k_{F}^{-1}$.

The impurity-dominated transport behavior at low temperatures can be described in terms of an ensemble $\mathcal{I}$ of distributions of a large number of impurities. For every distribution of impurities, the corresponding oneelectron model Hamiltonian is

$$
H=H_{0}+\sum_{a} v_{a}(\mathbf{x}),
$$

where $H_{0}$ is the unperturbed Hamiltonian, $a$ labels impurities (or defects), and $v_{a}(\mathbf{x})$ is the scattering potential due to the impurity (or defect) located at position $\mathbf{x}_{a}$. In this Letter, we assume (like in Ref. [5]) that the Fermi level is well above the multibarrier potentials associated with the different layers, which corresponds to a freeelectron Hamiltonian $H_{0}$ in Eq. (1).

Transport properties will be described in terms of 
impurity-averaged functions. The impurity average is performed in a locally uniform way in each layer, and $s i$ multaneously over the whole system. Notice that the condition $l \gg D_{\text {at }}$, where $D_{\text {at }}$ is the distance between atomic planes, guarantees that conduction electrons propagate in an "effective locally homogeneous medium" and allows us to view multilayers as the juxtaposition, in one dimension, of locally homogeneous layers. For example, the average density of impurities $n_{\mathrm{imp}}(z)$ is constant in each layer.

Furthermore, the short range and spin dependence of the impurity potentials in Eq. (1) allows us to choose zero-range potentials

$$
v_{a}(\mathbf{x})=\lambda_{a}(\hat{\boldsymbol{\sigma}}) \delta\left(\mathbf{x}-\mathbf{x}_{a}\right),
$$

with coupling strength

$$
\lambda_{a}(\hat{\boldsymbol{\sigma}})=w_{a}+j_{a} \hat{\mathbf{M}}_{a} \cdot \hat{\boldsymbol{\sigma}}
$$

where $\hat{\boldsymbol{\sigma}}$ stands for the Pauli vector spin operator (which accounts for spin-dependent scattering), $\hat{\mathbf{M}}_{a}$ is a unit vector in the direction of magnetization of the respective magnetic layer, and $w_{a}$ and $j_{a}$ are constants (for the nonmagnetic layers $j_{a}=0$ ). Equations (1)-(3) correspond to those used in the quantum model of Ref. [5]; however, our solution to the model, implemented in real space, will be different.

The one-particle irreducible self-energy $\Sigma(\varepsilon)$ for the impurity-averaged functions can be calculated by isolating the one-particle irreducible parts of the diagrammatic expansion for the impurity-averaged total off-shell (reducible) $T$ matrix $\langle T(\varepsilon)\rangle_{\mathcal{I}}$, which is obtained from the solution to the operator equation

$$
T(\varepsilon)=\mathcal{V}+\mathcal{V} G_{0}(\varepsilon) T(\varepsilon),
$$

after the impurity average is applied term by term in the resulting Dyson series. In Eq. (4), $\mathcal{V}=\sum_{a} v_{a}$ is the total potential acting on the electron and $G_{0}(\varepsilon)$ is the unperturbed one-particle propagator at energy $\varepsilon$. The dilute limit (that is, low concentration of impurities) restricts the irreducible self-energy to be given by the same sum of diagrams that defines the one-site off-shell $T$ matrix

$$
t_{a}(\varepsilon)=v_{a}+v_{a} G_{0}(\varepsilon) t_{a}(\varepsilon) .
$$

The resulting self-energy is local, namely, it is diagonal in the position representation (the actual degree of nonlocality is determined by the range of the potentials) with diagonal elements

$$
\Sigma(z)=n_{\mathrm{imp}}(z) t(z),
$$

where $t(z)$ is the real-space diagonal element of the onesite $T$-matrix at position $z$ :

$$
t(z)=\lambda(z)\left\{1-\lambda(z) \operatorname{tr}\left[G_{0}(\varepsilon)\right]\right\}^{-1},
$$

with $\lambda(z)$ being the coupling strength at position $z$ and $\operatorname{tr}\left[G_{0}(\varepsilon)\right]$ being the trace of the unperturbed one-particle propagator [10].

The ensuing impurity-averaged retarded one-particle propagator $\langle G(\varepsilon)\rangle_{\mathcal{I}}$ is then given by the Dyson formula, and its matrix elements in real space $\left\langle\mathbf{x}|G(\varepsilon)| \mathbf{x}^{\prime}\right\rangle$ exhibit an exponential decay with a mean free path $l(z)$, such that

$$
\Delta(z)=-\operatorname{Im}[\Sigma(z)]=\frac{\varepsilon_{F}}{k_{F} l(z)}
$$

(where, for transport properties at low temperature, the electron's energy is the Fermi energy $\left.\varepsilon=\varepsilon_{F}=\hbar^{2} k_{F}^{2} / 2 m\right)$. The real part of the self-energy can be absorbed as a redefinition of the energy reference level. The Dyson formula implies that the "reduced" one-dimensional oneparticle propagator $\mathcal{G}\left(z, z^{\prime}\right)$, which is defined via restoration of translational invariance in the plane of the layers:

$$
\left\langle\mathbf{k}_{\|}, z|G(\varepsilon)| \mathbf{k}_{\|}{ }^{\prime}, z^{\prime}\right\rangle=\frac{\hbar^{2}}{2 m} \delta_{\mathbf{k}_{\|}, \mathbf{k}_{\|}{ }^{\prime}} \mathcal{G}\left(z, z^{\prime}\right),
$$

satisfies the differential equation

$$
\left[\frac{d^{2}}{d z^{2}}+k^{2}(z)\right] \mathcal{G}\left(z, z^{\prime}\right)=\delta\left(z-z^{\prime}\right)
$$

where $k(z)$ is a complex wave number or propagation constant, which is given by

$$
k(z)=\left[\bar{k}^{2}+i k_{F} / l(z)\right]^{1 / 2},
$$

in terms of the "reduced" one-dimensional momentum:

$$
\bar{k}=\left(k_{F}^{2}-k_{\|}^{2}\right)^{1 / 2} .
$$

Equation (10) has already been proposed, but within the framework of a different approach, by Vedyayev, Dieny, and Ryzhanova [11].

Let us now consider an arbitrary $N$-layered system and introduce the following notation. Each layer will be identified with a subscript $j$, with $j=1, \ldots, N$ : layer $\mathcal{L}_{j}=\left[z_{j-1}, z_{j}\right]$, of thickness $a_{j}=z_{j}-z_{j-1}$, will have a local propagation constant $k_{j}$ and a mean free path $l_{j}$. Then, the resolution $z=a_{j} u_{j}+z_{j-1}$, for $z$ in $\mathcal{L}_{j}$, permits the identification $z \equiv\left(j, u_{j}\right)$, which leads to a "layerindex notation"; for example, $\mathcal{G}\left(z, z^{\prime}\right)=\mathcal{G}_{j, j^{\prime}}\left(u_{j}, u_{j^{\prime}}\right)$, for $z \in \mathcal{L}_{j}$ and $z^{\prime} \in \mathcal{L}_{j^{\prime}}$. The main goal of the transport theory of multilayered structures is to predict the size effects, i.e., the dependence of transport properties with respect to the different length scales $a_{j}$. Quasiclassical size effects can be conveniently expressed in terms of the set of dimensionless size parameters $\Lambda_{j}=a_{j} / l_{j}$, if the effect of multibarrier potentials is neglected.

For a finite system confined to a region of size $L$ by an infinite potential wall, Dirichlet boundary conditions are required to account for external size effects. However, when $L \gg l_{j} \gg k_{F}^{-1}$ (for all $j$ ), size effects become asymptotically independent of any external boundary conditions, except for the detailed behavior of internal fields near the boundaries. In this Letter, we will con- 
sider only internal size effects, as for most multilayered structures $L \gg l_{j}$ (sandwich structures are an exception as their total thickness is usually comparable with the mean free paths). Therefore, we will replace Dirichlet boundary conditions by outgoing boundary conditions, as if the system were effectively infinite. The resulting retarded Green's function is

$$
\mathcal{G}_{j, j^{\prime}}\left(u_{j}, u_{j^{\prime}}\right)=\frac{1}{2 i \bar{k}} \exp \left[i \bar{k}\left|z-z^{\prime}\right|-\frac{t}{2} \phi\left(u_{j}, u_{j^{\prime}}\right)\right],
$$

where $t$ is a dimensionless variable defined by $t=k_{F} / \bar{k}=$ $(\cos \theta)^{-1}$, with $\theta$ being the angle of propagation of a quasiclassical electron with respect to the $z$ axis (and $\left.k_{z}=\bar{k}\right)$, and

$$
\phi\left(u_{j}, u_{j^{\prime}}\right)=\Lambda_{j>} u_{j>}-\Lambda_{j_{<}} u_{j_{<}}+\alpha_{j_{<}, j_{>}-1}
$$

with $j_{<}\left(j_{>}\right)$being the smaller (larger) of $j$ and $j^{\prime}$, and $\alpha_{j_{<}, j_{>}-1}=\sum_{j=j_{<}}^{j_{>}-1} \Lambda_{j}$. In Eq. (13), the limit $k_{F} l_{j} \gg 1$, for all $j$, has been used in order to arrive at a quasiclassical propagator; Eq. (11) shows that this propagator is valid for most of the effective transport range, with an infrared lower bound of the order of $k_{F} / \sqrt{k_{F} l_{j}} \ll k_{F}$.

What is the connection between the Boltzmann and the Kubo approaches? A quasiclassical theory is based on a distribution function, whereas the Kubo formalism is based on a one-particle propagator or Green's function. A direct comparison of our approach with the one based on the Boltzmann equation shows that the quasiclassical distribution functions are linear functionals of the electric field $E(z)$, which are given, up to a constant, by

$\left.g^{ \pm}\left(z, k_{z}\right) \propto \int_{I_{ \pm}} d z^{\prime}\left\langle\left[\mathcal{A}\left(z, z^{\prime}\right)\right]^{2}\right\rangle_{\mathrm{av}}\right|_{\bar{k}=k_{z}} E\left(z^{\prime}\right)$,

where \pm refers to the sign of the $z$ component of the velocity the quasiclassical electrons, $I_{ \pm}$are the intervals to the left $\left(z^{\prime}<z\right)$ and to the right $\left(z^{\prime}>z\right)$ of point $z$, and $\mathcal{A}\left(z, z^{\prime}\right)$ is the one-dimensional reduced form [see Eq. (9)] of the density of states operator $A(\varepsilon)=i\left[G_{\mathrm{ret}}(\varepsilon)-G_{\mathrm{adv}}(\varepsilon)\right]$, whose square is spatially averaged over a distance of a few times $k_{F}^{-1}$ in the quasiclassical limit; the proportionality constant in Eq. (15) depends upon the normalization chosen for the distribution functions. Equation (15) shows that quasiclassical electrons at position $z$ propagating in the positive (negative) $z$ direction correspond to all the electrons propagating from points $z^{\prime}$ to the left (right) of the final point $z$.

So far, we have shown that the Kubo formalism yields a quasiclassical description of transport in metallic multilayers and found the explicit link between the two approaches. In the remainder of this Letter, we will consider two additional novel aspects of transport in these structures: the treatment of interfaces and of spatial dispersion.
Interface scattering is the most delicate aspect in a transport theory of multilayers. For example, an increased density of scatterers near the interfaces may lead to a breaking of the basic assumption that impurities scatter independently, that is, that quantum interference effects are negligible. However, a complete analysis of this problem is beyond the scope of this Letter, where we only intend to show the existence of a simple and unified framework, based on the Kubo formula, for the treatment of both bulk and interface scattering. Such a unified framework can be developed if we assume that an electron is free to propagate over the whole system with small potential differences at the interfaces, but with a huge potential barrier at the outer boundaries; correspondingly, the transmission of an electron through a region of interfacial disorder can be modeled with the addition of a thin "interface layer." Therefore, we will assume that interface scattering can also be represented with the Hamiltonian of Eq. (1), by treating interfaces as additional thin layers $\mathcal{S}_{j_{s}}$, with layer thicknesses $a_{j_{s}}^{(s)}$ and characteristic interface-local mean free paths $l_{j_{s}}^{(s)}$, in addition to the "bulk layers" $\mathcal{L}_{j_{b}}$, with thicknesses $a_{j_{b}}$ and mean free paths $l_{j_{b}}$. The same treatment, but starting directly from the Boltzmann equation, has been proposed by Johnson and Camley [8].

Even though, in this Letter, we subscribe to the description of interface scattering with additional interface layers, we would like to emphasize that it naturally admits the following interpretation in terms of the concepts used in the Boltzmann equation approach. In effect, one could go one step further and replace the real interface regions by infinitely thin interfaces with "internal structure," which is a good approximation as we can safely assume that $a_{j_{s}}^{(s)} \ll a_{j_{b}}$. Then, the exponential decay of the one-particle propagator through a given interface layer $\mathcal{S}_{j_{s}}$ yields an effective "coherent transmission coefficient" $T_{j_{s}}^{(s)}(t)$ :

$$
T_{j_{s}}^{(s)}(t)=\exp \left(-t \Lambda_{j_{s}}^{(s)}\right),
$$

with $\Lambda_{j_{s}}^{(s)}=a_{j_{s}}^{(s)} / l_{j_{s}}^{(s)}$, which represents the fraction of electrons transmitted (probability of transmission) across a given interface. In this quasiclassical picture of geometric electron propagation, the complementary fractions $\left[1-T_{j_{s}}^{(s)}(t)\right]$ represent scattering at the interface layer, which, when the thickness $a_{j_{s}}^{(s)}$ is neglected, is interpreted as "diffuse scattering" (in this Letter, we have not considered multibarrier effects, which would otherwise be accounted for via reflection coefficients). Thus, the coefficients $T_{j_{s}}^{(s)}(t)$ have the same physical interpretation as the ones required in a quasiclassical theory of multilayers with diffusive interfaces in order to match boundary conditions at the level of the distribution function, as first introduced by Carcia and Suna [3] as a generalization of the specularity parameters of the Fuchs- 
Sondheimer theory [2]. However, our transmission coefficients, unlike the ones used by Carcia and Suna and in all the subsequent papers dealing with the quasiclassical approach, are not constant parameters but depend upon the angle of incidence $\theta$ of the electrons on the interface [with $t=(\cos \theta)^{-1}$ ]; they favor the passage of electrons with nearly normal incidence and suppress exponentially the contribution from electrons moving nearly parallel to the interfaces. For example, assuming that $l_{j_{s}}^{(s)} \ll l_{j_{b}}$ (for all $j_{s}$ and $j_{b}$ ) to further reduce the "internal structure" of the interface layers, the global in-plane conductivity of a binary superlattice in our approach is formally identical to the one given by Carcia and Suna [3], but now with an angle-dependent transmission coefficient $T^{(s)}(t)=e^{-t \Lambda^{(s)}}$. This interpretation of the transmission coefficients is hardly surprising in a quasiclassical theory which adopts the treatment of interfaces first introduced by Johnson and Camley [8]. However, we should emphasize again that a fundamental difference exists between the outer boundaries and the interfaces, and that, correspondingly, the angle-dependent reflection coefficients proposed in the Fuchs-Sondheimer theory of thin films are of a very different nature [12]; moreover, the angledependent transmission coefficients corresponding to superlattice potentials, as discussed, for example, by Hood and Falicov [13], refer to the scattering by potential barriers (superlattice potentials), which makes them very different from the ones due to interfacial disorder ("diffuse" scattering).

The main consequence of this new treatment of interfaces is that it enhances the contribution of interfaces to the total resistivity and, in order to fit experimental results, it requires mean free paths which are smaller than the ones used in the usual Boltzmann approach and closer to the ones used in the quantum approach. In fact, this new choice of mean free paths eliminates the objections raised against the quasiclassical approach [5]: it is not the quasiclassical approach itself but the usual treatment of interfaces that is flawed.

Finally, this Letter presents, for the first time, a two-point formalism for multilayered structures. For experiments with a uniform external electric field (as follows from translational invariance in the plane of the layers), the electrical linear response of the system is given by a diagonal two-point conductivity tensor $\boldsymbol{\sigma}\left(z, z^{\prime}\right)=\boldsymbol{\sigma}\left(\mathbf{k}_{\|}=0, z ; \mathbf{k}_{\|}{ }^{\prime}=0, z^{\prime}\right)$, with components $\sigma^{(\perp)}\left(z, z^{\prime}\right)=\sigma_{z z}\left(z, z^{\prime}\right)$ for current perpendicular to the plane of the layers (CPP) and $\sigma^{(\|)}\left(z, z^{\prime}\right)=\sigma_{x x}\left(z, z^{\prime}\right)$ for current in the plane of the layers (CIP). The Kubo formula in real space leads to the following two-point conductivity functions:

$$
\sigma^{(r)}\left(z, z^{\prime}\right)=\frac{3 C_{D}}{4} F^{(r)}\left(\phi\left(u_{j}, u_{j^{\prime}}\right)\right)
$$

where $\phi\left(u_{j}, u_{j^{\prime}}\right)$ is given by Eq. (14), $C_{D}=n e^{2} / m v_{F}$ (with $n$ being the density of conduction electrons; alternatively, $C_{D}$ is the ratio between the bulk conductivity $\sigma_{j}$ and the local mean path $l_{j}$ of any layer $\mathcal{L}_{j}$ ), $F^{(\|)}(x)=E_{1}(x)-E_{3}(x), F^{(\perp)}(x)=2 E_{3}(x)$, and $E_{n}(x)$ is the exponential integral function of order $n$. Notice that the conductivity is anisotropic as a result of the layering. The resulting linear transport theory, based on Eq. (17), is nonlocal, with a characteristic length scale given by the mean free path. This nonlocality, called spatial dispersion, could be probed via electric fields which vary over that length scale.

Summarizing, in this Letter we presented a linear transport theory of multilayered structures that stems from the quantum-statistical Kubo formula: It yields a quasiclassical and nonlocal description of transport phenomena, with a novel treatment of scattering at interfaces.

We would like to thank Professor A. Fert and Dr. S. Zhang for insightful discussions and suggestions. This work was supported in part by the Office of Naval Research Grant No. N00014-91-J-1695.

[1] M. N. Baibich, J. M. Broto, A. Fert, F. Nguyen Van Dau, F. Petroff, P. Etienne, G. Creuzet, A. Friederich, and J. Chazelas, Phys. Rev. Lett. 61, 2472 (1988).

[2] K. Fuchs, Proc. Cambridge Philos. Soc. 34, 100 (1938); E. H. Sondheimer, Adv. Phys. 1, 1 (1952).

[3] P. F. Carcia and A. Suna, J. Appl. Phys. 54, 2000 (1983).

[4] R. E. Camley and J. Barnás, Phys. Rev. Lett. 63, 664 (1989).

[5] P. M. Levy, S. Zhang, and A. Fert, Phys. Rev. Lett. 65, 1643 (1990); Phys. Rev. B 45, 8689 (1992).

[6] R. Kubo, J. Phys. Soc. Jpn. 12, 570 (1957).

[7] A. Barthélémy and A. Fert, Phys. Rev. B 43, 13124 (1991).

[8] B. L. Johnson and R. E. Camley, Phys. Rev. B 44, 9997 (1991).

[9] G. D. Mahan, Many-Particle Physics (Plenum, New York, 1990), 2nd ed., pp. 259-266 and 601-634.

[10] S. Doniach and E. H. Sondheimer, Green's Functions for Solid State Physicists (Benjamin, Reading, MA, 1974), pp. $78-81$.

[11] A. Vedyayev, B. Dieny, and N. Ryzhanova, Europhys. Lett. 19, 329 (1992).

[12] J. M. Ziman, Electrons and Phonons (Clarendon, Oxford, 1960), pp. 456-460; R. F. Greene and R. W. O'Donnell, Phys. Rev. 147, 599 (1966); S. B. Soffer, J. Appl. Phys. 38, 1710 (1967).

[13] R. Q. Hood and L. M. Falicov (unpublished). 